\title{
A voluntary use of insecticide-treated cattle can eliminate African sleeping sickness
}

\author{
Kira Crawforda ${ }^{\text {, Aaleah Lancaster }}{ }^{\mathrm{a}}$, Hyunju Oha and Jan Rychtár ${ }^{\mathrm{b}}$ \\ aDepartment of Mathematics and Computer Sciences, Bennett College, Greensboro, NC, USA; ${ }^{\mathrm{b}}$ Department of \\ Mathematics and Statistics, The University of North Carolina at Greensboro, Greensboro, NC, USA
}

\begin{abstract}
African sleeping sickness is a vector-borne disease caused by the parasite Trypanosoma brucei. It is transmitted by tsetse flies and one of the most effective strategies to combat the disease is the use of insecticide-treated cattle (ITC). In this paper, we present a gametheoretical model, in which individual farmers choose their own level of ITC in order to maximize their own benefits, effectively balancing the cost of ITC and the risk of their cows contracting the disease. We find that even when the usage of ITC is strictly voluntary, the optimal ITC usage will eliminate the disease when the cost of ITC is not prohibitively large. This is in a sharp contrast with similar scenarios of vaccination games where a voluntary vaccination never eliminates a disease entirely.
\end{abstract}

\section{ARTICLE HISTORY}

Received 20 July 2015

Accepted 29 September 2015

\section{KEYWORDS}

Game theory; vector control; African sleeping sickness; insecticide

\section{Introduction}

African sleeping sickness (African Trypanosomiases) is a vector-borne disease caused by the parasite Trypanosoma brucei (Simarro, Jannin, \& Cattand, 2008). The disease is fatal in humans if left untreated (Coleman \& Welburn, 2004; Davis, Aksoy, \& Galvani, 2011) and the treatment of livestock has been hindered by drug resistance (Bourn, Grant, Shaw, \& Torr, 2005; Hargrove, Omolo, Msalilwa, \& Fox, 2000; Kajunguri et al., 2014). The disease is transmitted by tsetse flies and the most effective strategy to combat the disease is to eliminate the vectors (Rowlands et al., 2001). The use of insecticide-treated cattle (ITC) as baits for the tsetse flies is an effective vector control method causing little environmental damage (Hargrove, Ouifki, Kajunguri, Vale, \& Torr, 2012; Kabayo, 2002; Peter, Van den Bossche, Penzhorn, \& Sharp, 2005; Vale \& Torr, 2005). The original ITC protocol was to treat the whole body of the adult cattle but an application of insecticide to only legs where the flies bite most often (Torr et al., 2001) and to the ears where ticks accumulate, improved the cost and efficacy of tsetse control (Torr, Maudlin, \& Vale, 2007). Still, to be effective, the ITC technique should be applied to a large area to prevent re-infestation by the controlled region (Torr \& Vale, 2011). It requires participation and cooperation by a large number of farmers who are encouraged to control tsetse themselves rather than relying on governments (Kajunguri et al., 2014). 
Game-theoretical models of individuals facing a decision to either do a disease-preventive action (such as to vaccinate or to use ITC) or to risk contracting the disease have become increasingly common (Fenichel, Horan, \& Hickling, 2010; Reeling \& Horan, 2015; Wang \& Hennessy, 2015). Game theory has been applied to vaccination against major public health threats, including smallpox (Bauch, Galvani, \& Earn, 2003), measles (Shim et al., 2012b), rubella (Shim, Kochin, \& Galvani, 2009), childhood diseases (Bauch, 2005), influenza (Galvani, Reluga, \& Chapman, 2007) and many others. There is no human vaccine against African Trypanosomiases (La Greca \& Magez, 2011; Tabel, Wei, \& Bull, 2013). The ITC technique is not a vaccination and does not prevent cattle from contracting the disease (Vale, Mutika, \& Lovemore, 1999). Still, ITC can be seen as a disease-preventive action as the use of ITC reduces the incidence of the disease in the population and it can thus be modelled in a similar way as vaccination (Bauch \& Earn, 2004).

From the game theoretic perspective, an individual takes an action (such as to use ITC) that will maximize personal pay-off, taking into account the disease incidence and risk of infection which is determined by actions taken by the rest of the population (Shim, Chapman, Townsend, \& Galvani, 2012a). In theory, individuals are driven by self-interests rather than by the interest of the group (Shim, Meyers, \& Galvani, 2011). A usual outcome is that as the frequency of preventive actions increases, the incentive to take such an action decreases because of indirect protection by other protected individuals (Bauch \& Earn, 2004). This makes the eradication of a disease very difficult and practically impossible, even when the cost of the preventive action is very low (Geoffard \& Philipson, 1997).

In this paper, we follow a general approach of previous game theoretical models such as Bauch and Earn (2004) and apply the approach to a situation where individuals choose to use ITC to reduce the tsetse vector prevalence. Unlike in previously studied vaccination games, the use of ITC does not prevent the cows contracting the disease (Kajunguri et al., 2014), but rather helps to reduce the number of tsetse flies and consequently helps to increase the number of healthy cows. Thanks to this indirect but large benefit, our model shows that even when the use of ITC is strictly voluntary, the optimal level of protection yields the level needed for tsetse elimination, i.e. effectively achieving the herd immunity that was not possible in vaccination games.

\section{Mathematical model}

\subsection{Transmission model with ITC}

Our model relies heavily on the results and analysis of Kajunguri (2013) and Kajunguri et al. (2014). In Kajunguri et al. (2014), the authors present a SIR model involving all the variables related to tsetse vector control by ITC in a multi-host population. A slightly modified model for one host only (cattle) is shown in Figure 1 and described in this section. The notation and model parameters are summarized in Table 1.

The adult cattle population is divided into three classes, susceptible $(S)$, infectious $(I)$ and recovered $(R)$, whereas the tsetse population is divided into two classes, susceptible $\left(S_{V}\right)$ and infectious $\left(I_{V}\right)$. To include ITC in the model, we split the cattle population into treated (with superscript $t$ ) and untreated with (with superscript $u$ ). The ITC does not prevent the tsetse fly from biting but each fly that bites or touches a treated cow dies within a few hours (Vale, Mutika, \& Lovemore, 1999). It means that $I$ and $R$ classes are also divided into treated and untreated 
Table 1. Notations and parameter values, adapted from Kajunguri et al. (2014).

\begin{tabular}{llll}
\hline & Meaning & Value & Source \\
\hline$\Lambda$ & Host recruitment rate & 22 & WHO (2012) \\
$\mu$ & Host natural mortality & .00055 & WHO (2012) \\
$a$ & Biting rate & .25 & Coleman and Welburn (2004) \\
$\beta$ & Prob. of transmission from fly to cattle & .62 & Rogers (1988) \\
$\alpha$ & Prob. of transmission from cattle to fly & .065 & Rogers (1988) \\
$T_{H}$ & Incubation period in host & 7 days & Coleman and Welburn (2004) \\
$T_{V}$ & Incubation period in flies & 18 days & Coleman and Welburn (2004) \\
$\sigma$ & Mortality of infected hosts & .006 & Davis et al. (2011) \\
$g$ & Host recovery rate & .014 & Davis et al. (2011) \\
$V$ & Rate of immunity loss in hosts & 1 & Coleman and Welburn (2004) \\
$B_{V}$ & Tsetse birth rate & 1440 & Davis et al. (2011) \\
$\Lambda_{V}$ & Tsetse recruitment rate & Equation (5) & Kajunguri et al. (2014) \\
$\mu_{V}$ & Tsetse natural mortality rate & .03 & Rogers (1988) \\
$m$ & Tsetse additional mortality due to ITC & .57 & Torr et al. (2001) \\
$m_{V}$ & Tsetse mortality rate with ITC & Equation (4) & Kajunguri et al. (2014) \\
$d^{-1}$ & Duration of insecticide efficacy & 28 days & Torr et al. (2007) \\
$\psi$ & Proportion of cattle being treated & variable & \\
$\pi$ & Stable proportion of treated cattle & $\psi /(\psi+d)$ & \\
$\lambda$ & Force of infection in hosts & Equation (14) & \\
$\lambda_{V}$ & Force of infection in flies & Equation (15) & \\
$C$ & Normalized cost of ITC per cow per day & variable & \\
$x$ & Percentage of cattle owned by a focal farmer & variable & \\
\hline
\end{tabular}
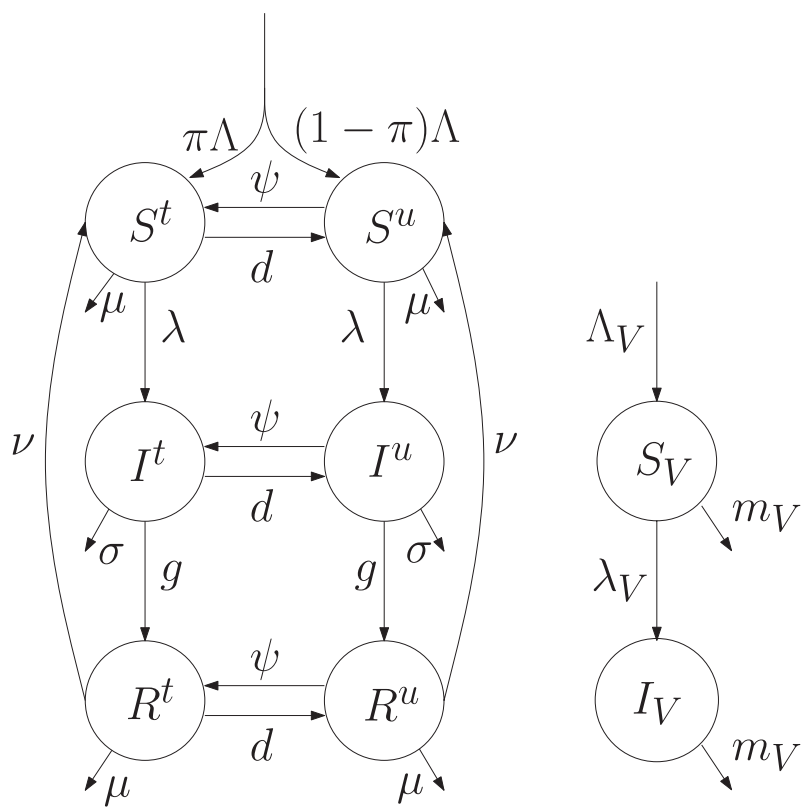

Figure 1. Compartmental model of African sleeping sickness transmission in cattle host and Tsetse flies, adapted from Kajunguri et al. (2014). There is a time lag due to the incubation period and so at time $t$, $\lambda\left(t-T_{H}\right) S\left(t-T_{H}\right)$ of cattle leave the susceptible and enters the infections class; here $\lambda\left(t-T_{H}\right)$ is the force of infection at time $t-T_{H}$ and $S\left(t-T_{H}\right)$ is the susceptible population at time $t-T_{H \cdot}$. A similar situation occurs with tsetse flies where the force of infection is $\lambda_{V}$ and the incubation period is $T_{V}$. 


$$
\begin{gathered}
S=S^{t}+S^{u}, \\
I=I^{t}+I^{u}, \\
R=R^{t}+R^{u} .
\end{gathered}
$$

We assume the cattle is treated at rate $\psi$, thus moving from untreated classes $S^{u}, I^{u}$ or $R^{u}$ to the treated classes, $S^{t}, I^{t}$ or $R^{t}$. The insecticide effect lasts on average for $1 / d$ days after which the cattle moves from the treated classes, $S^{t}, I^{t}$ or $R^{t}$ to the untreated classes $S^{u}, I^{u}$ or $R^{u}$.

The tsetse flies feed on the adult cattle. The feeding rate is $a$, i.e. each fly takes a new blood meal on average every 1 / $a$ days. During the blood meal, the disease is transmitted from an infected cow to a fly with probability $\alpha$ and from an infected fly to a cow with probability $\beta$. As in Kajunguri et al. (2014), the time delays $T_{H}$ and $T_{V}$ are representing the incubation period in the host and tsetse vector populations, respectively.

The cattle recruitment rate $\Lambda$; the rate is given by a combination of the immigration and reaching adult size. A newly recruited cow is assumed to be susceptible. However, unlike Kajunguri et al. (2014), we assume that the proportion $\pi=\psi /(\psi+d)$ of the recruited cattle is treated, i.e. the proportion of treated versus untreated newly recruited cattle is $\psi / d$. This assumption is the only deviation from the model described in Kajunguri et al. (2014) and we do it in order to make the flow chart in Figure 1 more symmetric which allows us to get an analytical solution and postpone the use of computation by one step. The assumption can be easily satisfied by treating the newly recruited cattle.

The mortality of the tsetse flies is given by

$$
m_{V}=\mu_{V}+a m \frac{S^{t}+I^{t}+R^{t}}{N}
$$

where $\mu_{V}$ is the natural mortality, $N=S+I+R$ is the total number of cattle and $\left(S^{t}+I^{t}+R^{t}\right) / N$ is the probability a flies bites a treated cow and $m$ is the additional mortality due to the insecticides.

The recruitment rate of tsetse vectors is derived in Kajunguri et al. (2014) as

$$
\Lambda_{V}=\frac{B_{V}}{a+m_{V}}\left(1-\exp \left(-a-m_{V}\right)\right)
$$

where $B_{V}$ is the tsetse birth rate.

The flow chart on Figure 1 then yields the following equations

$$
\begin{gathered}
\frac{\mathrm{d}}{\mathrm{d} t} S^{u}=(1-\pi) \Lambda+\nu R^{u}+d S^{t}-(\psi+\mu) S^{u}-\lambda\left(t-T_{H}\right) S^{u}\left(t-T_{H}\right) \\
\frac{\mathrm{d}}{\mathrm{d} t} I^{u}=\lambda\left(t-T_{H}\right) S^{u}\left(t-T_{H}\right)+d I^{t}-(\psi+g+\sigma) I^{u} \\
\frac{\mathrm{d}}{\mathrm{d} t} R^{u}=g I^{u}+d R^{t}-(\psi+\mu+v) R \\
\frac{\mathrm{d}}{\mathrm{d} t} S^{t}=\pi \Lambda+\psi S^{u}+\nu R^{t}-(d+\mu) S^{t}-\lambda\left(t-T_{H}\right) S^{t}\left(t-T_{H}\right)
\end{gathered}
$$




$$
\begin{gathered}
\frac{\mathrm{d}}{\mathrm{d} t} I^{t}=\psi I^{u}+\lambda\left(t-T_{H}\right) S^{t}\left(t-T_{H}\right)-(d+g+\sigma) I^{t} \\
\frac{\mathrm{d}}{\mathrm{d} t} R^{t}=\psi R^{u}+g I^{t}-(d+\mu+v) R^{t} \\
\frac{\mathrm{d}}{\mathrm{d} t} S_{V}=\Lambda_{V}-\exp \left(-m_{V} T_{V}\right) \lambda_{V}\left(t-T_{V}\right) S_{V}\left(t-T_{V}\right)-m_{V} S_{V} \\
\frac{\mathrm{d}}{\mathrm{d} t} I_{V}=\exp \left(-m_{V} T_{V}\right) \lambda_{V}\left(t-T_{V}\right) S_{V}\left(t-T_{V}\right)-m_{V} I_{V}
\end{gathered}
$$

where

$$
\begin{aligned}
& \lambda=a \beta \frac{I_{V}}{N}, \\
& \lambda_{V}=a \alpha \frac{I}{N}
\end{aligned}
$$

are the forces of infection. As we will later see from (21), $\lambda$ plays a critical role in determining the equilibrium value of $N$, the total count of the cattle. In turn, $N$ is critical in evaluating the farmer's benefits as seen in (20).

\subsection{Game-theoretical model}

Let us assume that an individual farmer owns a proportion $x$ of all of the cattle in a given region that is large enough to prevent an immediate tsetse re-introduction from regions with no tsetse control (Torr \& Vale, 2011). Let $\psi_{0}$ be the average ITC usage among other farmers in the area. We want to optimize $\psi_{f}$, the level of ITC usage of the focal farmer. The average usage of ITC in the population is given by

$$
\psi=x \psi_{f}+(1-x) \psi_{0} .
$$

If there are $N$ cattle in total, the focal farmer will have to treat $\psi_{f} x N$ of the existing cattle as well as proportion $x \pi$ of the newly recruited cattle (assuming the recruitment of cattle is uniform among the farmers). When the system (6)-(13) is in equilibrium, $N \mu=\Lambda$ and thus the total number of treated cattle per day, $N_{f}^{t}$, is given by $x N\left(\psi_{f}+\pi \mu\right)$. Assuming $x$ is relatively small, we get

$$
N_{f}^{t} \approx x N\left(\psi+\mu \frac{\psi_{0}}{\psi_{0}+d}\right)
$$

Let $C$ be the normalized cost of treatment of one cow per day (assuming benefits of having such a cow are equal to 1$)$. We can then evaluate $E\left(\psi_{f}, \psi_{0}\right)$, the focal farmer's net benefits of using strategy $\psi_{f}$ in the population where everybody else uses strategy $\psi_{0}$ by

$$
E\left(\psi_{f}, \psi_{0}\right)=x N-C N_{f}^{t}
$$




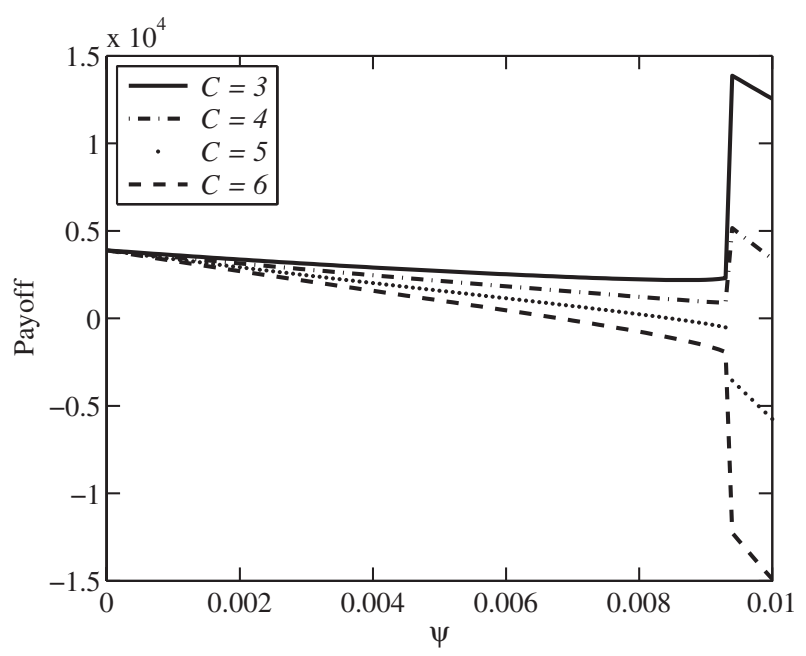

Figure 2. Dependence of pay-off $E(\psi, \psi)$ on the ITC level of treatment $\psi$ for different relative costs of treatments $C$. Parameter values as in Table 1 . Since the farmer uses strategy $\psi$ as everybody else in the population, the value of $x$ is irrelevant in this case.

$$
\begin{aligned}
& =x N\left(1-C \psi-C \mu \frac{\psi_{0}}{\psi_{0}+d}\right) \\
& =x N\left(1-C x \psi_{f}-C(1-x) \psi_{0}-C \mu \frac{\psi_{0}}{\psi_{0}+d}\right) .
\end{aligned}
$$

\section{Results}

When we rewrite system (6)-(11) for $S, I, R$, we get exactly the system (4)-(8) from Kajunguri et al. (2014) (for the model formally without ITC).

Following Kajunguri et al. (2014), we have

$$
N=\frac{G \lambda+H}{D \lambda+F}
$$

where the force of infection $\lambda$ satisfies

$$
\lambda\left(a_{0} \lambda^{2}+b_{o} \lambda+c_{0}\right)=0
$$

and the auxiliary variables $a_{0}, b_{0}, c_{0}, A, B, D, F, G, H$ and the basic reproduction number $R_{0}$ are given by

$$
R_{0}^{2}=\frac{\exp \left(-m_{V} T_{V}\right)}{m_{V}^{2}} \cdot \frac{\alpha a^{2} \beta \mu \Lambda_{V}}{\Lambda(g+\sigma)}
$$

and 


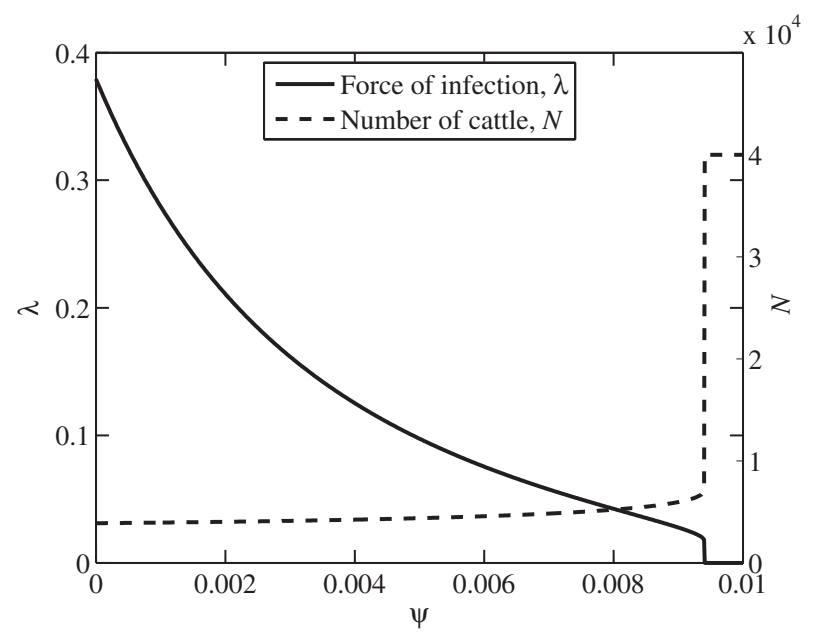

Figure 3. Dependence of force of infection $\lambda$ and the total number of cattle $N$ on the ITC level of treatment $\psi$ (this is independent of the relative costs of treatments C). Parameter values as in Table 1.
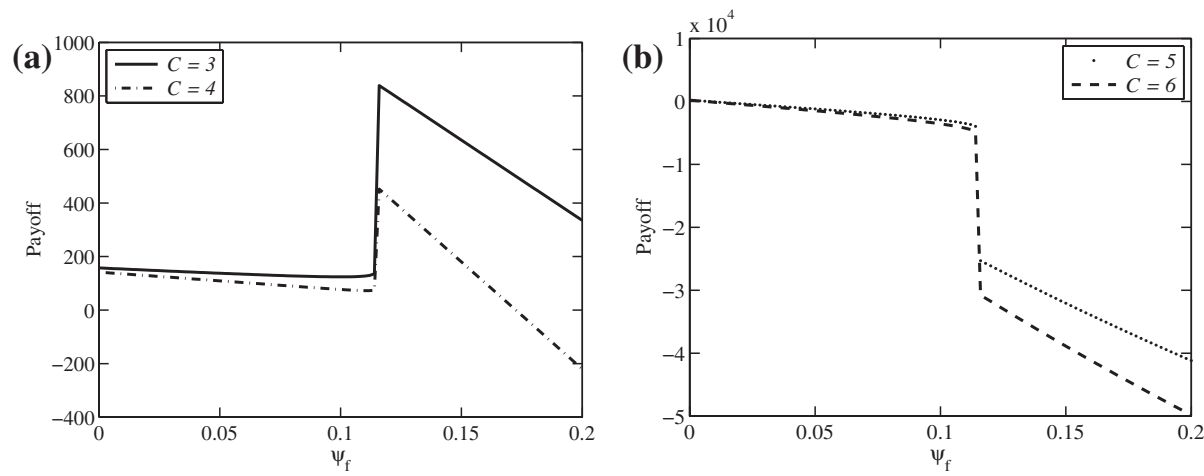

Figure 4. Dependence of pay-off $E\left(\psi_{f}, \psi_{0}\right)$ on the individual ITC level of treatment $\psi_{f}$ for different relative costs of treatments $C, \psi_{0}=.003, x=.05$, and other parameter values as in Table 1 . The jump occurs exactly when the average level of ITC usage $\psi$ is at the critical value yielding the eradication of the disease.

$$
\begin{gathered}
a_{0}=G m_{V}\left(A \alpha a B+G m_{V}\right) \\
b_{0}=A H \alpha a B m_{V}+2 H G m_{V}^{2}-A \alpha a^{2} \Lambda_{V} B D \beta \\
c_{0}=\Lambda m_{V}^{2}(g+\sigma)\left(1-R_{0}^{2}\right) \\
A=\exp \left(-m_{V} T_{V}\right) \\
B=\Lambda(\mu+v) \\
D=g \mu+\sigma(\mu+v)
\end{gathered}
$$




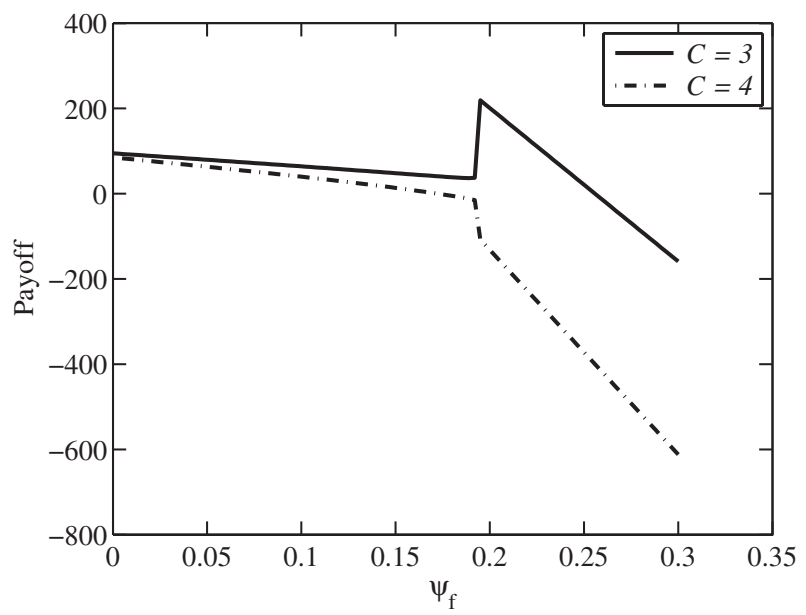

Figure 5. Dependence of pay-off $E\left(\psi, \psi_{0}\right)$ on the individual ITC level of treatment $\psi_{f}$ for different relative costs of treatments $C, \psi_{0}=.003, x=.03$, and other parameter values as in Table 1 . The jump occurs exactly when the average level of ITC usage $\psi$ is at the critical value yielding the eradication of the disease. However, the value at which the jump occurs is not always optimal. Compare to Figure 4.

$$
\begin{gathered}
F=\mu(\nu+\mu)(g+\sigma) \\
G=\Lambda(g+v+\mu) \\
H=\Lambda(\nu+\mu)(g+\sigma) .
\end{gathered}
$$

Substituting (21) into (20) then yields the explicit formula for the focal farmer's net benefits, $E\left(\psi_{f}, \psi_{0}\right)$.

First, assume that everybody uses the same level of ITC. Figure 2 shows the pay-off $E(\psi, \psi)$ for various costs of treatment $C$, depending on the ITC level $\psi$. Note the sharp jump at payoffs that happens exactly at the value of $\psi$ where $\lambda$ becomes 0 and where the number of cattle jumps from a relatively low to relatively large value, see Figure 3 . For relatively small values of $C$, such a critical value of $\psi$ is optimal from the population perspective (as it yields the highest pay-off for the population as a whole) and it is also a value of ITC usage that eradicates the tsetse flies and the disease. The disease is eradicated at the same value of $\psi$ even when the cost of ITC is large. However, for such a large $C$, the critical level of ITC is no longer optimal and the pay-off (from the population perspective) is the highest when no ITC is used at all.

We note that while the critical value $\psi$ is relatively small (less than $1 \%$ ), the cost of using ITC may still be too large for some poorer farmers or some farmers may simply choose not to use it. In order to complete the analysis, we thus need to consider a focal farmer using $\psi_{f}$ in the population of other farmers using $\psi_{0}$ on average and determine the optimal strategy for such a farmer. Figure 4 shows that when $C$ is sufficiently small (below the threshold from Figure 2), the optimal ITC usage for a focal farmer is exactly such that the overall average ITC usage is at the critical value, resulting in the elimination of the disease. However, Figure 4(b) illustrates that when the cost of using ITC is large, the optimal usage for the individual farmer is 0 . The same is true when the cost of using ITC is relatively small, but the farmer does not own enough cattle, see Figure 5. 


\section{Conclusions}

Following Bauch and Earn (2004), we provided a game-theoretical model to study the optimal usage of ITC to combat African sleeping sickness. Our analysis is built on the transmission model of sleeping sickness by Kajunguri et al. (2014) that studied the efficiency of ITC. For numerical simulations, we focused on the restricted application of ITC, the formulae and results for whole body applications are similar.

Not surprisingly, our results indicate that the optimal usage of ITC depends on the cost of ITC. From the population perspective (for example, when the usages is mandated/subsidied by the government), when the cost of ITC is not prohibitive, the optimal usage of ITC yields a complete eradication of the disease; otherwise, when the cost of ITC treatments is above a certain threshold, it is optimal not to use ITC at all.

We found that eradication of African sleeping sickness by voluntary usage of ITC is possible (again, when the cost of treatment is not prohibitively large). This finding is in stark contrast with previous work on voluntary disease prevention, see for example (Bauch, 2005; Bauch \& Earn, 2004; Geoffard \& Philipson, 1997). As discussed in Hargrove, Torr, $\&$ Kindness (2003) and Kajunguri et al. (2014), the real-world success of ITC depends on many factors such as the size and shape of the controlled region and the number or density of treated cattle. Nevertheless, application of ITC can indeed lead to reduction of the incidence of sleeping sickness cases (Magona \& Walubengo, 2011).

The main reason for such a difference between findings of our analysis and the previous work such as Bauch and Earn (2004) lies in the highly bimodal distribution of the benefits. In the presence of the disease (i.e. when ITC usage is not enough to eradicate it), the equilibrium level of cattle and consequently the farmer benefits are much smaller than when the ITC usage is at or above the eradication levels. We note that the same feature is present in the original Kajunguri et al. (2014) model from which we differed only in treating the newly recruited cattle directly. When the cost of ITC is not large and when the farmer has a sufficiently large population of cattle that his decision can significantly affect the average usage level, the individual interests of the farmer aligns well with the interest of the population and the disease can be eradicated.

We note that our model can be further improved. We used a simple pay-off function (20) that can be made more realistic by including exact benefits of owning a cow (a per day benefit as well as per cow benefit) as well as exact cost of the treatment (set-up costs and per cow cost). Moreover, the added benefits of ITC include tick control (Bourn et al., 2005; Torr, Maudlin, \& Vale, 2007; Vale \& Torr, 2005) are not included in our model. For the sake of simplicity, we also used only the single-host transmission model from Kajunguri et al. (2014) while multi-host models (including humans) are also available and we did not include the benefits of smaller disease incidence among humans in our model either. Nevertheless, we speculate that adding extra benefits or extending the model for the multi-host situation will not qualitatively change the results. The situation may be different if tsetse flies start to develop resistance against the treatment. As suggested in Magona and Walubengo (2011), a combination of different treatments is likely to minimize the occurrence and spread of the resistant parasites.

Also, individuals can have different perceptions of the real situation (Poletti, Ajelli, \& Merler, 2011) and can base their decision also on different social aspects (Xia \& Liu, 2013). Providing proper information may thus play an important role in disease eradication. As 
noted in Kajunguri et al. (2014), the model presented here does not work when cattle populations are migratory and travel widely through tsetse infested country. Nevertheless, game-theoretical models, in general, can still capture the interplay between disease prevalence, protective action coverage and individual behaviour (Bauch, 2005) and we can argue that since the real benefits to an individual farmer exceeds the benefits considered in our simple model, the optimal usage of ITC yields an eradication of the disease even when the ITC is voluntary.

\section{Acknowledgements}

The authors also wish to thank the anonymous reviewers for their suggestions that helped to improve the manuscript.

\section{Disclosure statement}

No potential conflict of interest was reported by the authors.

\section{References}

Bauch, C. T. (2005). Imitation dynamics predict vaccinating behaviour. Proceedings of the Royal Society B: Biological Sciences, 272, 1669-1675.

Bauch, C. T., \& Earn, D. J. (2004). Vaccination and the theory of games. Proceedings of the National Academy of Sciences of the United States of America, 101, 13391-13394.

Bauch, C. T., Galvani, A. P., \& Earn, D. J. (2003). Group interest versus self-interest in smallpox vaccination policy. Proceedings of the National Academy of Sciences, 100, 10564-10567.

Bourn, D., Grant, I., Shaw, A., \& Torr, S. (2005). Cheap and safe tsetse control for livestock production and mixed farming in Africa. Aspects of Applied Biology, 75, 1-12.

Coleman, P. G., \& Welburn, S. C. (2004). Are fitness costs associated with resistance to human serum in Trypanosoma brucei rhodesiense? Trends in Parasitology, 20, 311-315.

Davis, S., Aksoy, S., \& Galvani, A. (2011). A global sensitivity analysis for African sleeping sickness. Parasitology, 138, 516-526.

Fenichel, E. P., Horan, R. D., \& Hickling, G. J. (2010). Bioeconomic management of invasive vectorborne diseases. Biological Invasions, 12, 2877-2893.

Galvani, A. P., Reluga, T. C., \& Chapman, G. B. (2007). Long-standing influenza vaccination policy is in accord with individual self-interest but not with the utilitarian optimum. Proceedings of the National Academy of Sciences, 104, 5692-5697.

Geoffard, P.-Y., \& Philipson, T. (1997). Disease eradication: Private versus public vaccination. The American Economic Review, 87, 222-230.

Hargrove, J., Omolo, S., Msalilwa, J., \& Fox, B. (2000). Insecticide-treated cattle for tsetse control: The power and the problems. Medical and Veterinary Entomology, 14, 123-130.

Hargrove, J., Torr, S., \& Kindness, H. (2003). Insecticide-treated cattle against tsetse (Diptera: Glossinidae): What governs success? Bulletin of Entomological Research, 93, 203-217.

Hargrove, J. W., Ouifki, R., Kajunguri, D., Vale, G. A., \& Torr, S. J. (2012). Modeling the control of trypanosomiasis using trypanocides or insecticide-treated livestock. PLoS Neglected Tropical Diseases, 6, e1615.

Kabayo, J. P. (2002). Aiming to eliminate tsetse from Africa. Trends in Parasitology, 18, 473-475.

Kajunguri, D. (2013). Modelling the control of tsetse and African trypanosomiasis through application of insecticides on cattle in Southeastern Uganda (PhD thesis), Stellenbosch: Stellenbosch University.

Kajunguri, D., Hargrove, J. W., Ouifki, R., Mugisha, J., Coleman, P. G., \& Welburn, S. C. (2014). Modelling the use of insecticide-treated cattle to control tsetse and Trypanosoma brucei rhodesiense in a multi-host population. Bulletin of Mathematical Biology, 76, 673-696. 
La Greca, F., \& Magez, S. (2011). Vaccination against trypanosomiasis: Can it be done or is the trypanosome truly the ultimate immune destroyer and escape artist? Human Vaccines, 7, 12251233.

Magona, J., \& Walubengo, J. (2011). Mass-treatment and insecticide-spraying of animal reservoirs for emergency control of Rhodesiense sleeping sickness in Uganda. Journal of Vector Borne Diseases, 48, 105-108.

Peter, R., Van den Bossche, P., Penzhorn, B. L., \& Sharp, B. (2005). Tick, fly, and mosquito control lessons from the past, solutions for the future. Veterinary Parasitology, 132, 205-215.

Poletti, P., Ajelli, M., \& Merler, S. (2011). The effect of risk perception on the 2009 H1N1 pandemic influenza dynamics. PLoS One, 6, e16460.

Reeling, C. J., \& Horan, R. D. (2015). Self-protection, strategic interactions, and the relative endogeneity of disease risks. American Journal of Agricultural Economics, 97, 452-468.

Rogers, D. (1988). A general model for the African trypanosomiases. Parasitology, 97, 193-212.

Rowlands, G., Leak, S., Mulatu, W., Nagda, S., Wilson, A., \& d'Ieteren, G. (2001). Use of deltamethrin pour-oninsecticide for the control of cattle trypanosomosis in the presence of high tsetse invasion. Medical and Veterinary Entomology, 15, 87-96.

Shim, E., Chapman, G. B., Townsend, J. P., \& Galvani, A. P. (2012a). The influence of altruism on influenza vaccination decisions. Journal of The Royal Society Interface. page rsif20120115. doi: http://dx.doi.org/10.1098/rsif.2012.0115

Shim, E., Grefenstette, J. J., Albert, S. M., Cakouros, B. E., \& Burke, D. S. (2012b). A game dynamic model for vaccine skeptics and vaccine believers: measles as an example. Journal of Theoretical Biology, 295, 194-203.

Shim, E., Kochin, B., \& Galvani, A. (2009). Insights from epidemiological game theory into genderspecific vaccination against rubella. Mathematical Biosciences and Engineering: MBE, 6, 839-854.

Shim, E., Meyers, L. A., \& Galvani, A. P. (2011). Optimal H1N1 vaccination strategies based on self-interest versus group interest. BMC Public Health, 11(Suppl 1), S4. doi: http://dx.doi.org/10.1186/1471-2458-11-S1-S4. Retrieved from http://www.biomedcentral. com/1471-2458/11/S1/S4 >

Simarro, P. P., Jannin, J., \& Cattand, P. (2008). Eliminating human African trypanosomiasis: Where do we stand and what comes next? PLoS Medicine, 5, e55.

Tabel, H., Wei, G., \& Bull, H. J. (2013). Immunosuppression: Cause for failures of vaccines against African trypanosomiases. PLoS Neglected Tropical Diseases, 7, e2090.

Torr, S., Maudlin, I., \& Vale, G. (2007). Less is more: Restricted application of insecticide to cattle to improve the cost and efficacy of tsetse control. Medical and Veterinary Entomology, 21, 53-64.

Torr, S., Wilson, P., Schofield, S., Mangwiro, T., Akber, S., \& White, B. (2001). Application of DNA markers to identify the individual-specific hosts of tsetse feeding on cattle. Medical and Veterinary Entomology, 15, 78-86.

Torr, S. J., \& Vale, G. A. (2011). Is the even distribution of insecticide-treated cattle essential for tsetse control? Modelling the impact of baits in heterogeneous environments. PLoS Neglected Tropical Diseases, 5, e1360.

Vale, G., Mutika, G., \& Lovemore, D. (1999). Insecticide-treated cattle for controlling tsetse flies (Diptera: Glossinidae): Some questions answered, many posed. Bulletin of Entomological Research, 89, 569-578.

Vale, G., \& Torr, S. (2005). User-friendly models of the costs and efficacy of tsetse control: Application to sterilizing and insecticidal techniques. Medical and Veterinary Entomology, 19, 293-305.

Wang, T., \& Hennessy, D. A. (2015). Strategic interactions among private and public efforts when preventing and stamping out a highly infectious animal disease. American Journal of Agricultural Economics, 97, 435-451.

WHO. (2012). Uganda: Country health profile. Retrieved from http://www.who.int/gho/countries/ uga.pdf?ua $=1$

Xia, S., \& Liu, J. (2013). A computational approach to characterizing the impact of social influence on individuals' vaccination decision making. PLoS One, 8, e60373. 